\title{
Die Linke zwischen internen Konflikten, der ersten Koalition im Westen, Niederlagen im Osten und dem Ramelow-Effekt
}

\author{
Hendrik Träger
}

\section{$1 \quad$ Einleitung}

Die ambivalente Situation der Linken im Herbst 2019 - also zwei Jahre nach der Bundestagswahl 2017 - lässt sich anhand von zwei Zitaten verdeutlichen: „Dieser Sieg gibt uns Schwung für die ganze Partei“, sagte die Parteivorsitzende Katja Kipping (zit. nach Locke und Becker 2019, S. 3) nach der Thüringer Landtagswahl im Oktober 2019. Unter Führung von Ministerpräsident Bodo Ramelow war Die Linke erstmals stärkste politische Kraft in einem Bundesland geworden. Lediglich sieben Wochen zuvor hatten Antje Feiks und Thomas Dudzek (zit. nach Debski 2019, S. 1), die damals Vorsitzende und Geschäftsführer des sächsischen Landesverbandes gewesen sind, die „Gesamtpartei [...] vor einer existenziellen Herausforderung [gesehen; H.T.]. Es geht um nicht weniger als die Zukunft der Linken insgesamt." Im September 2019 hatte Die Linke sowohl in Sachsen als auch in Brandenburg ihren Stimmenanteil fast halbiert und das schlechteste beziehungsweise zweitschlechteste Landtagswahlergebnis seit 1990 verbuchen müssen.

Diese elektoralen Abstürze trafen die Partei in einer zwiespältigen Situation: Zum einen war nur wenige Wochen zuvor in Bremen die erste Koalition unter Beteiligung der Linkssozialisten in einem westdeutschen Bundesland gebildet worden, sodass die Partei erstmals gleichzeitig an vier Regierungen beteiligt war. Zum anderen war Die Linke bei der Europawahl im Mai 2019 mit einem Stimmenanteil von 5,5\% auf den niedrigsten Wert seit ihrer Gründung zwölf

\footnotetext{
H. Träger $(\bowtie)$

Institut für Politikwissenschaft, Universität Leipzig, Leipzig, Deutschland E-Mail: hendrik.traeger@uni-leipzig.de
} 
Jahre zuvor respektive - die PDS mitberücksichtigend - den zweitniedrigsten Wert ihrer Geschichte gerutscht. Außerdem hatte Sahra Wagenknecht (zit. nach Fried 2019a, S. 5) ${ }^{1}$ im März 2019 bekannt bekanntgegeben, im Herbst nicht wieder für das Amt der Bundestagsfraktionsvorsitzenden zu kandidieren, und ihre Entscheidung mit den gesundheitlichen Problemen als ,eine[r] direkte[n] Folge des extremen Stresses, den ich in den letzten Jahren hatte“, begründet. In diesem Statement spiegeln sich die teilweise heftigen Auseinandersetzungen zwischen dem auch in den eigenen Reihen umstrittenen „Star der Partei“ (Küpper 2016, S. 4) und großen Teilen der Linken wider.

Sowohl diese Personalentscheidung als auch die skizzierten Wahlergebnisse sind wichtige Wegmarken bei der Entwicklung der Linken seit der Bundestagswahl am 24. September 2017. Mit diesen Punkten und weiteren Aspekten beschäftigt sich der vorliegende Beitrag und knüpft damit an die ähnlich konzipierten Analysen von Gero Neugebauer und Richard Stöss seit der Bundestagswahl 1998 an (vgl. Neugebauer 2011; Neugebauer und Stöss 1999, 2003, 2007, 2015). Besonders relevant sind hier die Studien seit 2005, bei denen zunächst das Wahlbündnis Linkspartei(.PDS) und danach Die Linke im Mittelpunkt standen. Um die einschlägigen Studien mit der folgenden Analyse zu verknüpfen, werden zunächst wichtige Befunde von Neugebauer und Stöss aufgegriffen und auf dieser Grundlage Leitfragen generiert:

- Anlässlich der Bundestagswahl 2005 konstatierten Neugebauer und Stöss (2007, S. 162), dass „die Partei (...) von einer Mehrheit ihrer Wähler als Mittel zum Protest genutzt wurde." Acht Jahre später beobachteten die Autoren ,eine gewisse Attraktivität auf ethnozentrisch gesinntes Protestpotenzial" (Neugebauer und Stöss 2015, S. 164). Wurde Die Linke auch im September 2017 vorrangig als Ausdruck des Protestes gewählt? Oder hat sich die Motivlage ihrer Wählerschaft gewandelt?

- Nach der Wahl 2009 kritisierte Gero Neugebauer (2011, S. 172) auch, dass Die Linke „entweder neben oder hinter den Debatten her“ trabe und „kein Gesicht [präsentiert; H.T.], das sich in der Wahrnehmung der Öffentlichkeit mit einem bestimmten Politikfeld verbindet." Infolgedessen würde die Partei mit ihren Anliegen weder bei den Medien noch in der Öffentlichkeit Interesse wecken und deshalb in Umfragen verlieren oder zumindest stagnieren. Lässt sich Ähnliches auch gegenwärtig beobachten?

\footnotetext{
${ }^{1}$ Ein Video zur Pressekonferenz von Sahra Wagenknecht und Dietmar Bartsch ist abrufbar unter: https://www.sahra-wagenknecht.de/de/article/2851.gemeinsam-streiten-f\%C3\%BCreinen-linken-aufbruch.html.
} 
- Ebenfalls nach der Bundestagswahl 2009 konstatierte Neugebauer (2011, S. 167) - der schon von Robert Michels beobachteten Tendenz in sozialistischen Parteien folgend - eine „Dominanz der Fraktion“, weil „kein strategisches Zentrum in der eigentlichen Parteistruktur" existiere. Ist Die Linke weiterhin hauptsächlich eine Fraktionspartei? Oder haben sich die Machtverhältnisse zwischen Fraktion und Partei verschoben?

- Bei der Bundestagswahl 2013 habe Die Linke, argumentieren Neugebauer und Stöss (2015, S. 170), „den Zenit ihrer Entwicklung bereits überschritten“. Die forcierte Westausdehnung sei nicht wirklich erfolgreich gewesen; bei der Linken handele es sich weiterhin ,faktisch um eine Ostpartei mit Brückenköpfen im westlichen Einzugsgebiet“ (ebd., S. 166). Trifft das immer noch zu?

Um diese Fragen beantworten zu können, werden zunächst die Situation der Linken vor der Bundestagswahl 2017 skizziert (Abschn. 2), die programmatischen Schwerpunkte und das Personaltableau im Wahlkampf beschrieben sowie die Ergebnisse des Urnengangs analysiert (Abschn. 3). Anschließend richtet sich der Fokus auf die Entwicklung der Partei seit September 2017. Dabei wird sowohl auf die Debatten über die Positionen von Sahra Wagenknecht in der Flüchtlingspolitik und die Sammlungsbewegung, aufstehen ' (Abschn. 4) als auch auf die Ergebnisse der Linken bei den Landtagswahlen und der Europawahl (Abschn. 5) eingegangen. Im Fazit werden die wichtigsten Befunde zusammengefasst und ein Ausblick auf die nächsten Jahre gewagt (Abschn. 6).

\section{Die Linke vor der Bundestagswahl 2017: eine ambivalente Ausgangslage}

Infolge der Bildung einer Großen Koalition nach der Bundestagswahl 2013 stellte Die Linke erstmals die größte Oppositionsfraktion, sodass der damalige Fraktionsvorsitzende Gregor Gysi die Funktion des Oppositionsführers übernahm. Der „talentierte(...) Rhetoriker und charmante(...) Plauderer“ (Ehrenstein 2015, S. 6) war geradezu die Idealbesetzung, um der Regierung im Parlament gelegentlich auf unterhaltsame Weise - Paroli zu bieten. Allerdings kündigte er im Juni 2015 seinen Rücktritt zur Mitte der Legislaturperiode an. Mit ihm zog sich die letzte der drei „großen Integrations- und Galionsfiguren der LinKen“ (Oppelland und Träger 2014, S. 232) aus der ersten Reihe zurück.

Bei der Regelung der Nachfolge entschied sich die Fraktion für ,eines der verrückteren Politikexperimente der bundesdeutschen Geschichte" (Wiedmann-Schmidt 2016a, S.34): Mit Sahra Wagenknecht und Dietmar Bartsch wurde eine 
Doppelspitze gebildet, die keineswegs als politisches „Traumpaar“ (Kosfeld 2015, S. 12) gelten kann, sondern die verschiedenen Strömungen innerhalb von Partei und Fraktion widerspiegelt. Während Wagenknecht, die seit 2014 mit Oskar Lafontaine verheiratet ist, als ehemalige Vertreterin der Kommunistischen Plattform (KPF) dem orthodoxen Lager der Linken zugeordnet werden kann, gehört Bartsch zu den Reformern und den innerparteilichen Kritikern von Lafontaine.

Wagenknecht entwickelte sich für Partei und Fraktion als Segen und Fluch zugleich: Einerseits ist die „wortgewandte, zuspitzende Diskutantin“ (Jesse und Lang 2012, S. 361) eine medienwirksame Repräsentantin der Linken, die relativ schnell ähnlich hohe Beliebtheitswerte wie ihr Vorgänger erreichte (vgl. Hilmer und Gagné 2018, S. 399). Andererseits bringt die „Frau, die in kein Schema passt“" (Hujer 2015, S. 40), häufig die eigenen Genossinnen und Genossen gegen sich auf. So kritisierte sie unmittelbar vor den drei Landtagswahlen im März 2016 die Flüchtlingspolitik der Bundesregierung, erklärte, „dass Kapazitäten nicht unbegrenzt sind“ (Wagenknecht 2016), und warnte „,vor ,Parallelwelten““ (Wiedmann-Schmidt 2016c, S. 32). Solche Wortmeldungen sind nicht mit den Beschlüssen der Linken kompatibel und verleiteten innerparteiliche Kritikerinnen und Kritiker dazu, Wagenknecht in die Nähe der AfD zu rücken. In diesem Zusammenhang attestierte wiederum Oskar Lafontaine (2016, S. 31) seiner Partei einen ,schlechte[n] Stil“.

Die Situation eskalierte letztlich so weit, dass auf dem Parteitag im Mai 2016 ein Aktivist Wagenknecht eine Torte ins Gesicht warf; außerdem wurden Flyer mit dem Aufdruck „Torten für Menschenfeinde“ (Wyssuwa 2016, S. 2) verteilt. Im weiteren Verlauf des Jahres nahm auch die Kritik aus den Reihen der Bundestagsfraktion zu; mit Jan van Aken forderte ein Abgeordneter sogar den Rücktritt der Vorsitzenden (vgl. Küpper 2016, S. 4; Wiedmann-Schmidt 2016b, S. 38).

Die Diskussionen über die Positionen in der Flüchtlings- und Asylpolitik resultierten insbesondere aus den deutlichen Verlusten der Linken bei einigen Landtagswahlen und dem gleichzeitigen Aufstieg der AfD. Die Rechtspopulisten erhielten in großem Umfang Stimmen von ehemaligen Wählerinnen und Wählern der Linken und entwickelten sich damit zu einer ernstzunehmenden Konkurrenz um Protestwählerinnen und -wähler (vgl. Träger 2018b, S. 4 f.). Dies ist ein wichtiger Grund dafür, dass Die Linke 2016 in Mecklenburg-Vorpommern ,ihr historisch schwächstes Ergebnis“ (Koschkar \& Nestler 2017, S. 39) verbuchen musste und bei der Berliner Abgeordnetenhauswahl ,in einigen Stadtrandbereichen von der AfD überholt" (Niedermayer 2017a, S. 48) wurde. Ein halbes Jahr später war sie im Saarland ,[n]eben den Piraten (...) die größte Verliererin der Wahl“" (Winkler 2018, S. 48). 
Zur elektoralen Bilanz der Linken in den vier Jahren der 18. Legislaturperiode des Bundestages (2013-2017) gehören aber auch einige Erfolge: In Brandenburg konnte 2014 die rot-rote Koalition trotz deutlicher Verluste der Regierungsparteien fortgesetzt werden (vgl. Niedermayer 2015). In Thüringen wurde mit Bodo Ramelow erstmals ein Politiker der Linken zum Ministerpräsidenten eines Bundeslandes gewählt; mit Rot-Rot-Grün gab es ein neues Koalitionsmodell in Deutschland (vgl. Oppelland 2015; Oppelland und Träger 2016). Nach der Berliner Abgeordnetenhauswahl 2016 verständigten sich SPD, Linke und Grüne auf ein gemeinsames Bündnis, sodass Die Linke zum ersten Mal in ihrer Geschichte gleichzeitig an drei Landesregierungen beteiligt war.

In den westdeutschen Bundesländern konnte die Partei - mit Ausnahme des Saarlandes (2017) - ihren Stimmenanteil entweder stabil halten oder ausbauen, schaffte es aber nur in Hessen, den beiden Stadtstaaten und dem Saarland in das Landesparlament. Im Mai 2017 scheiterte sie in Nordrhein-Westfalen - trotz ihrer „Erfolge in den Großstädten“ (Bajohr 2017, S. 626) - mit 4,9\% äußerst knapp an der Fünf-Prozent-Hürde und spielte vor der schleswig-holsteinischen Wahl ,in der landespolitischen Auseinandersetzung kaum eine Rolle“ (Knelangen 2017, S. 581).

Die anhaltenden Differenzen zwischen Ost und West spiegeln sich nicht nur in den Wahlergebnissen, sondern auch bei den Mitgliedern wider: Bundesweit schwankte die Rekrutierungsfähigkeit der Linken in den Jahren vor der Bundestagswahl 2017 zwischen 0,08 und 0,09\%; der Partei gehörten also acht oder neun von 10.000 parteibeitrittsberechtigten Personen an. In den neuen Ländern entwickelte sich der Wert rückläufig, war aber mit 0,24 bis $0,28 \%$ immer noch um ein Vielfaches höher als im Westen (Abb. 1). Obgleich Die Linke also weiterhin in Ostdeutschland wesentlich stärker verankert ist, sprach Tim Spier (2016, S. 201) zurecht nicht mehr von ,einer reinen ,Regionalpartei Ost ${ }^{\star}(\ldots)$ sondern vielmehr von einer bundesweiten Partei mit Hochburgen in Ostdeutschland.“

Mit Blick auf die Mitglieder der Linken ist wichtig, dass 2017 ungefähr zwei Drittel Männer waren und knapp ein Sechstel jünger als 35 Jahre alt war. Der letzte Wert hatte sich seit 2009 mehr als verdoppelt. Die Linke hatte inzwischen „proportional die meisten jungen Mitglieder“ (Klein et al. 2019, S. 87). Gleichwohl waren die Rentnerinnen und Rentner, die mehr als die Hälfte der Mitglieder stellten, im Vergleich zur Gesamtbevölkerung überproportional vertreten. Gleiches ist bei Menschen mit einem (Fach-)Hochschulabschluss, Auszubildenden respektive Studierenden sowie Arbeitslosen $\mathrm{zu}$ beobachten. Das Gegenteil traf auf Vollerwerbstätige sowie auf Menschen mit Hauptschulabschluss und mittlerer Reife zu (vgl. ebd., S. 89 f.). Mit $34 \%$ ist „der Anteil 


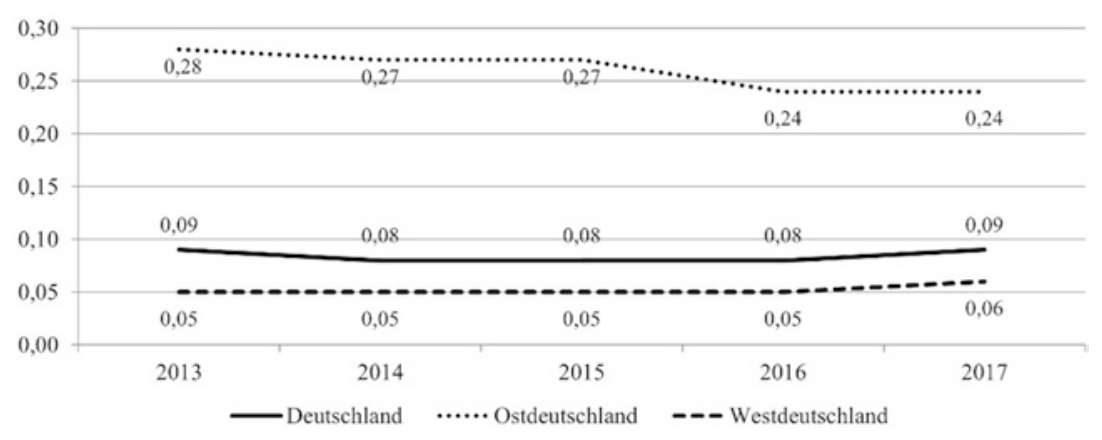

Abb. 1 Rekrutierungsfähigkeit der Linken in den Jahren 2013 bis 2017 (in Prozent). Lesebeispiel: Im Jahr 2013 gehörten 0,09\% aller Parteibeitrittsberechtigten der Linken an. (Quelle: Eigene Darstellung nach Daten von Oskar Niedermayer 2016, 2017b, 2018b, 2019)

derjenigen, die sich der Unterschicht oder der unteren Mittelschicht zugehörig fühlten, (...) deutlich höher als bei allen anderen Parteien, aber auch höher als in der Bevölkerung insgesamt“ (ebd., S. 94). Das spiegelt sich letztlich auch im Wahlprogramm wider (s. u.).

Obgleich Die Linke die stärkste Oppositionsfraktion im Bundestag war, konnte sie nicht von der Schwäche der Regierungsparteien profitieren, wie angesichts ausgewählter, Sonntagsfragen“ für den ARD-DeutschlandTrend und das ZDF-Politbarometer deutlich wird: Seit Herbst 2013 verharrten die Werte mit acht bis zehn Prozent auf dem Niveau des letzten Wahlergebnisses $(8,9 \%)$. Auch in der Zeit, als intensiv über die Asyl- und Integrationspolitik der Bundesregierung diskutiert wurde, gab es keine spürbaren Veränderungen. Selbst der Achtungserfolg ihres Kandidaten Christoph Butterwegge bei der Bundespräsidentenwahl im Februar $2017^{2}$ wirkte sich für Die Linke nicht positiv aus. Im Frühjahr 2017 rutschte die Partei zeitweise sogar auf sechs beziehungsweise sieben Prozent ab, was vor allem mit dem kurzzeitigen Schulz-Hype bei der SPD erklärt werden kann. Bis zur Bundestagwahl kamen die Linkssozialisten nicht über neun Prozent hinaus (Abb. 2). Die Ausgangssituation vor dem Urnengang war also keineswegs optimal, denn eine rechnerische Mehrheit für eine

\footnotetext{
${ }^{2}$ Das Ergebnis des parteilosen Armutsforschers Christoph Butterwegge lag 33 Stimmen über dem Sitzkontingent der Linken in der Bundesversammlung.
} 


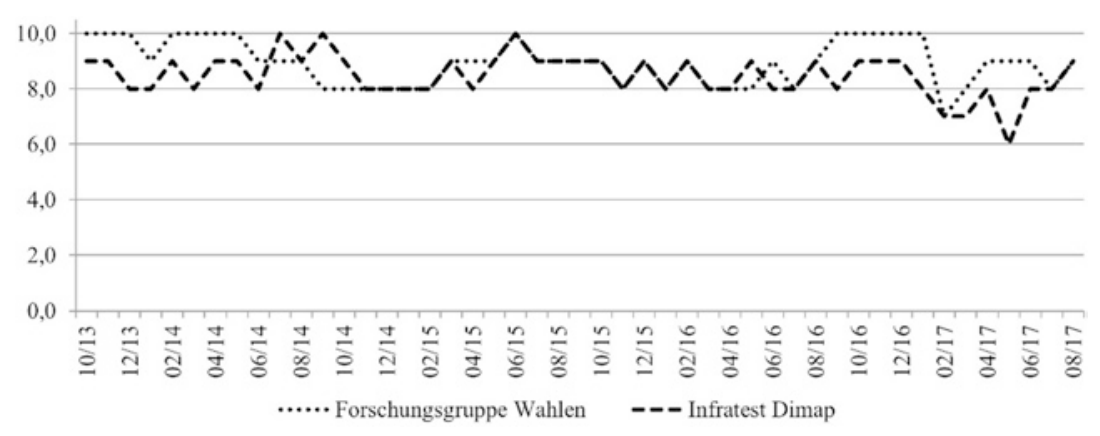

Abb. 2 Die Linke in den ,Sonntagsfragen“ vor der Bundestagswahl 2017 (in Prozent). Für die Monate mit mehreren ,Sonntagsfragen“ ist der Wert der jeweils letzten Umfrage abgebildet. (Quelle: Eigene Darstellung nach Daten von www.wahlrecht.de)

rot-rot-grüne Koalition als einzige Machtoption der Linken war - mit Ausnahme weniger Umfragen im Frühjahr 2017 - unrealistisch. Das wirkte sich auch auf den Wahlkampf aus, in dem die Partei „nur noch eine untergeordnete Rolle“ (Hilmer und Gagné 2018, S. 383) spielte.

\section{Die Bundestagswahl 2017: Gewinne im Westen und Verluste im Osten}

In den Wahlkampf zog Die Linke - trotz negativer Erfahrungen der PDS bei der Bundestagswahl 2002 (vgl. Neugebauer und Stöss 2003) - mit dem ungewöhnlichen Modell eines vierköpfigen Spitzenteams, das durch den damaligen Bundesgeschäftsführer Matthias Höhn unterstützt werden sollte: Als Spitzenkandidaten berief der Parteivorstand im Dezember 2016 Dietmar Bartsch und Sahra Wagenknecht. Diesem Duo wurden die wenige Monate zuvor ohne Gegenkandidaten wiedergewählten Parteivorsitzenden Katja Kipping und Bernd Riexinger zur Seite gestellt. Beiden war es seit 2012 gelungen, ,die Flügelkämpfe zwischen Fundamentalisten und Reformern zumindest nach außen einzudämmen“ (Niedermayer 2018a, S. 300). Zu dem Vorstandsbeschluss gehörte auch die folgende Regelung:

„Die Kommunikationsstrategie wird gemeinsam im Spitzenteam erarbeitet. Die politischen Entscheidungen zum Wahlprogramm sowie die politischen Entscheidungen nach der Wahl werden in enger Absprache mit den Spitzenkandidaten geführt und liegen bei der Partei und ihren Vorsitzenden“"(Die Linke 2016). 
Dieser Passus und die gesamte Konstruktion des Personaltableaus machen zum einen die Dominanz der Fraktion bei der öffentlichen Wahrnehmung der Linken und zum anderen die angespannte Beziehung zwischen den beteiligten Akteuren, wegen der offenbar eine schriftliche Fixierung der Zuständigkeiten erforderlich war, deutlich. Das sind ungünstige Voraussetzungen für einen harmonischen Wahlkampf aus einem Guss.

Auch die Aufstellung der einzelnen Landeslisten war umkämpft und einer Untersuchung des Berliner Instituts für Parlamentarismusforschung (Schüttemeyer und Höhne 2019, S. 30) zufolge - für „,39,0 Prozent der befragten Linke-Mitglieder (...) eher nicht (32,2 Prozent) bzw. gar nicht vorhersehbar $(6,8$ Prozent)“. Im Durchschnitt bewarben sich 2,3 Personen für einen Listenplatz; nur in der AfD und bei Bündnis 90/Die Grünen waren es noch mehr (vgl. ebd., S. 18). Ob dieser hohe Wert auf ein großes Interesse an einer politischen Karriere im Bundestag oder auf intensive Duelle um die sicheren Listenplätzen zurückzuführen ist, muss an dieser Stelle offen bleiben.

Im Juni 2017 verabschiedete Die Linke auf einem Parteitag ihr Wahlprogramm. In dessen Präambel bezeichnete die Partei den bevorstehenden Urnengang als „eine Richtungsentscheidung“ (Die Linke 2017, S. 7), bei der es um „ein sozialeres, nachhaltigeres Land, ein gerechtes Europa und eine friedlichere Welt" (ebd.) gehe. Angesichts der oben skizzierten sozioökonomischen Struktur der Mitglieder war es zu erwarten, dass sich die Linkssozialisten hauptsächlich für sozialpolitische Projekte wie eine Erhöhung des Mindestlohnes auf zwölf Euro, die Ablösung von Hartz IV durch ,eine bedarfsdeckende und sanktionsfreie Mindestsicherung in Höhe von 1050 EUR“ (ebd., S. 10), die Einführung einer solidarischen Mindestrente (1.050,- EUR), eine Vermögensteuer, und eine solidarische Gesundheitsversicherung einsetzten. Eine sofortige, steuerfinanzierte Angleichung der Renten in Ostdeutschland an das Niveau im Westen, das Ende von Auslandseinsätzen der Bundeswehr, das Verbot von Rüstungsexporten und eine Auflösung der NATO waren weitere bereits in früheren Programmen artikulierte Positionen. Mit der Bekämpfung von Fluchtursachen und der Integrationspolitik beschäftigte sich Die Linke (2017, S. 64-67, S. 97 f.) in vergleichsweise kurzen Abschnitten ihres Programmes, was angesichts der politischen Brisanz des Themas seit dem Spätsommer 2015 verwundert. Das lässt sich auch mit den innerparteilichen Differenzen hinsichtlich dieses Politikfeldes erklären.

Sowohl die inhaltlichen Positionen in der Außen- und Sicherheitspolitik als auch despektierliche Äußerungen der Spitzenkandidatin à la „Raute oder Zottelbart im Kanzleramt" (Wagenknecht 2017) mit Blick auf Angela Merkel und Martin Schulz sorgten dafür, dass der Parteitag „der letzte Sargnagel im 
rot-rot-grünen Projekt" wurde, wie Richard Hilmer und Jérémie Gagné (2018, S. 383) konstatieren. Eine Koalition aus SPD, Linken und Bündnis 90/Die Grünen wurde - einer Umfrage der Forschungsgruppe Wahlen (2017, S. 27) zufolge - ohnehin als die schlechteste aller möglichen Kombinationen beurteilt. Die gleiche Erhebung ergab, dass das Ansehen der Linken in der Bevölkerung seit 2013 deutlich gestiegen, aber immer ,noch mäßig negativ“ (ebd., S. 22) war. Bei den Spitzenkandidaten rangierte Wagenknecht - Bartsch wurde nicht berücksichtigt - leicht im positiven Bereich. Allerdings wurde ihr Wert bei allen Befragten nur von jenem für Alexander Gauland (AfD) unterboten. Auch die eigene Anhängerschaft stand ihr im Vergleich zu den Kombinationen bei den anderen Parteien reserviert gegenüber (vgl. ebd., S. 32).

Hinsichtlich der zugewiesenen Kompetenz lag Die Linke in allen Politikfeldern mit großem Abstand auf dem dritten oder vierten Platz. Lediglich bei der sozialen Gerechtigkeit kam sie mit $15 \%$ relativ nah an die Unionsparteien (21\%) heran, hatte aber weiterhin großen Abstand zur SPD (34\%). Programmatisch konnte die Partei also trotz eines Wahlkampfes mit starker „Policyzentrierung“ (Fitzpatrick 2018, S. 607) nicht überzeugen.

Ein erstaunliches Ergebnis der Vorwahlbefragung ist angesichts einer dezidierten „Abgrenzung zur amtierenden Kanzlerin“ (Fitzpatrick 2018, S. 617) im Online-Wahlkampf, dass $74 \%$ der Anhängerschaft der Linken Angela Merkel gute Arbeit attestierten; dieser Wert lag sogar knapp über dem Durchschnitt aller Parteien (vgl. Forschungsgruppe Wahlen 2017, S. 29). Wenn große Teile der Anhängerschaft der größten Oppositionspartei die Kanzlerin so positiv beurteilen, dann zeugt das davon, dass es der Linken nicht einmal im eigenen Lager gelang, sich als ,Regierung im Wartestand' zu präsentieren. Das sind ungünstige Voraussetzungen für einen erfolgreichen Wahlkampf.

Vor diesem Hintergrund ist es fast überraschend, dass Die Linke bei der Bundestagswahl 2017 ihren Zweitstimmenanteil von 8,6 auf 9,2\% leicht ausbauen und damit das zweitbeste Ergebnis ihrer Geschichte verbuchen konnte. Vor allem aufgrund der deutlich gestiegenen Wahlbeteiligung verzeichnete die Partei ein Plus von mehr als einer halben Million Wählerinnen und Wählern. Auch bei den gewonnenen Direktmandaten konnte sich die Partei von vier auf fünf geringfügig verbessern, ohne jedoch auch nur ansatzweise in die Nähe des Rekordwertes von 16 erfolgreichen Bewerberinnen und Bewerbern im Jahr 2009 zu kommen.

Trotz des Stimmenzuwachses verlor Die Linke ihre Position als drittstärkste Partei an die AfD und wurde sogar noch von der FDP überholt, sodass sie nunmehr - abermals knapp vor Bündnis 90/Die Grünen - lediglich fünfstärkste Fraktion im Bundestag ist und den Status als Oppositionsführerin verlor. 
Aufgrund der Mandatsverteilung im Parlament war ein rot-rot-grünes Bündnis nicht einmal mehr eine rechnerisch mögliche Option. Infolgedessen spielte Die Linke ebenso wie die AfD keine Rolle bei der Regierungsbildung und ist nur noch eine von vier Oppositionsfraktionen. Das wirkt sich negativ auf das mediale und öffentliche Interesse an der parlamentarischen Arbeit der Linken aus; es besteht gewissermaßen ein „Aufmerksamkeitsdefizit“ (Lang 2018, S. 56).

Mit Blick auf die regionale Verteilung der Zweitstimmen lässt sich konstatieren, dass Die Linke keineswegs mehr die „ostdeutsche Regionalpartei mit seltenen Erfolgen im Westen“ (Träger 2015, S. 68; ausführlicher: Oppelland und Träger 2014) wie früher ist. Zwar bilden weiterhin die neuen Länder und Berlin „eine geschlossene Gruppe mit den höchsten Zweitstimmenanteilen“ (Träger 2018a, S. 299); aber in Brandenburg, Mecklenburg-Vorpommern, Sachsen, Sachsen-Anhalt und Thüringen sowie in den östlichen Bezirken der Hauptstadt verlor Die Linke sowohl in absoluten Zahlen als auch hinsichtlich des Stimmenanteils erheblich an Wählerinnen und Wählern. Demgegenüber sind für alle westdeutschen Länder Zuwächse zwischen 1,3 und 3,4 Prozentpunkten zu verzeichnen (Tab. 1).

Der insgesamt höhere Zweitstimmenanteil ist also ausschließlich auf die größere Unterstützung im Westen zurückzuführen. Dieser Befund ist für eine Partei, die ihre Hochburgen traditionell in Ostdeutschland hat, ein Alarmsignal, denn frühere Wählergruppen gehen verloren. Das resultiert vor allem daraus, dass Die Linke ihren „Status als erste Adresse der Protestwähler“ (Lang 2018, S. 5) einbüßte und infolgedessen ,ein stetiger Transfer von der Linken zur AfD“ (ebd., S. 15) stattfand. Diese auf den ersten Blick kurios wirkenden Wählerwanderungen erklärt Eckhard Jesse (2018, S. 241) so:

„Die Protestwähler aus den Reihen der Partei Die Linke, die mittlerweile als etabliert gilt, jedenfalls in den neuen Bundesländern, sahen eine Alternative in der AfD.“

In diesem Zusammenhang dürfte es sich negativ ausgewirkt haben, dass Die Linke „den Themenkomplex Flüchtlinge, Integration und Zuwanderung im Wahlkampf schlicht nicht vorgesehen" habe, wie Jürgen P. Lang (2018, S. 32) beobachtete. Geradezu symbolisch dafür ist die vergleichsweise kurze Abhandlung des Politikfeldes im Wahlprogramm, auf die bereits oben hingewiesen wurde.

Hinsichtlich der Sozialstruktur der Wählerschaft fällt auf, dass Frauen (49\%) und Männer (51\%) ungefähr zu gleichen Teilen vertreten sind, mehr als die Hälfte $(57 \%)$ berufstätig ist und fast zwei Drittel 45 Jahre oder älter sind (vgl. Forschungsgruppe Wahlen 2017, S. 53 ff.). Gleichwohl ist ein 
Tab. 1 Die Linke bei der Bundestagswahl 2017

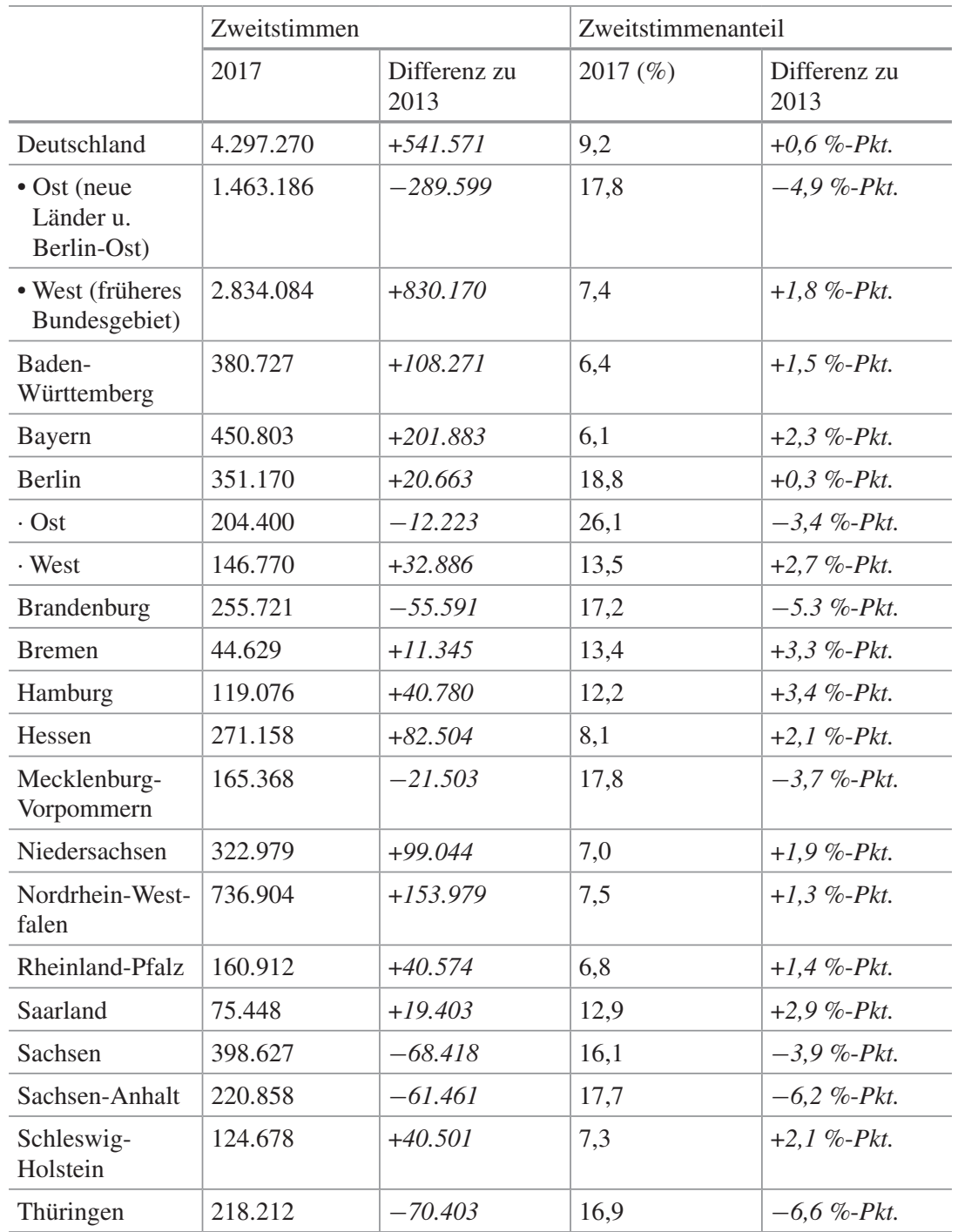

(Quelle: Eigene Darstellung und Berechnungen nach Daten des Bundeswahlleiters 2013, 2017) 
„Verjüngungsprozess“ (Hilmer und Gagné 2018, S. 390) zu beobachten, wobei es in den einzelnen Altersgruppen Unterschiede zwischen alten und neuen Ländern gibt. Diese Differenzen können auf ,das eher bodenständige Auftreten der Partei im Osten und das deutlich radikalere im Westen, womit sie junge Personen offenbar stärker anspricht“" (Jesse 2018, S. 235), zurückgeführt werden.

Aufgrund der gegensätzlichen Entwicklung der Zweitstimmenanteile mit teilweise erheblichen Verlusten im Osten und spürbaren Gewinne im Westen ist Die Linke im Bundestag „,keine primär ostdeutsche Partei mehr“, wie Richard Hilmar und Jérémie Gagné (2018, S. 391) feststellen. Den Recherchen von Jürgen P. Lang (2018, S. 22) zufolge stammt ,der größere Anteil an Abgeordneten aus den radikal-linken Westverbänden“. Diese Veränderungen dürften sich nachhaltig auf die Arbeit innerhalb der Fraktion und das äußere Erscheinungsbild der gesamten Partei auswirken.

\section{$4 \quad$ Parteiinterner Streit und Wagenknechts Bewegung, aufstehen'}

Die unterschiedliche Entwicklung in Ost und West ist nicht nur bei den Zweitstimmenanteilen, sondern auch bei den Parteimitgliedern zu beobachten (Tab. 2): Für die gesamte Partei ist kein einheitlicher Trend erkennbar. Der Mitgliederbestand blieb 2016 bei einem minimalen Rückgang von 79 Personen stabil, stieg ein Jahr später - vor allem wegen der häufig zu beobachtenden Mobilisierung im Kontext von Bundestagswahlen und aufgrund des „Trump-Effekt[s]“ (Niedermayer 2018b, S. 350) - deutlich auf 62.300 Genossinnen und Genossen an, um dann 2018 wieder geringfügig kleiner zu werden.

Mit Blick auf die Landesverbände sind jedoch eindeutige Tendenzen zu konstatieren: Während die Gebietsgliederungen im Westen und in Berlin seit 2015 jedes Jahr fast flächendeckend mehr Mitglieder als im Vorjahr verbuchen konnten, ist Die Linke im Osten kontinuierlich mit signifikanten Verlusten konfrontiert. Dadurch verschieben sich die Größenordnungen der Landesverbände. Im Jahr 2018 gab es in Nordrhein-Westfalen die meisten Linkssozialistinnen und Linkssozialisten, sodass erstmals in der Geschichte von PDS, Linkspartei und Die Linke ein westdeutscher Landesverband der größte der Partei ist. Der langjährige Spitzenreiter Sachsen liegt nur noch knapp auf dem zweiten Platz und dürfte auf dieser Position bald von Berlin abgelöst werden. Inwieweit das für das innerparteiliche Kräfteverhältnis relevant ist, wird sich zeigen. Wenn die fünf ostdeutschen Landesverbände deutlich weniger als die Hälfte der Mitglieder stellen, dürfte sich das negativ auf ihren Einfluss auswirken. Daran wird 
Tab. 2 Die Mitgliederentwicklung der Linken (2015-2018)

\begin{tabular}{l|l|l|l|l}
\hline & 2015 & 2016 & 2017 & 2018 \\
\hline Deutschland & 58.989 & 58.910 & 62.300 & 62.016 \\
\hline Baden-Württemberg & 2836 & 3134 & 3438 & 3532 \\
\hline Bayern & 2508 & 2457 & 3147 & 3417 \\
\hline Berlin & 7447 & 7508 & 7961 & 7861 \\
\hline Brandenburg & 6626 & 6212 & 6061 & 5802 \\
\hline Bremen & 481 & 507 & 579 & 624 \\
\hline Hamburg & 1307 & 1386 & 1577 & 1639 \\
\hline Hessen & 2525 & 2679 & 3029 & 3255 \\
\hline Mecklenburg-Vorpommern & 4034 & 3853 & 3725 & 3581 \\
\hline Niedersachsen & 2552 & 2742 & 3078 & 3112 \\
\hline Nordrhein-Westfalen & 6465 & 6703 & 7875 & 8183 \\
\hline Rheinland-Pfalz & 1594 & 1584 & 1633 & 1769 \\
\hline Saarland & 2114 & 2395 & 2465 & 2124 \\
\hline Sachsen & 8677 & 8284 & 8261 & 7988 \\
\hline Sachsen-Anhalt & 4044 & 3878 & 3776 & 3596 \\
\hline Schleswig-Holstein & 970 & 1019 & 1239 & 1298 \\
\hline Thüringen & 4767 & 4528 & 4444 & 4224 \\
\hline Ouelle: Er & Sal & & & \\
\hline
\end{tabular}

(Quelle: Eigene Darstellung nach Daten von Oskar Niedermayer (2016, 2017b, 2018b, 2019))

die Tatsache, dass Die Linke hinsichtlich der Rekrutierungsfähigkeit ,,immer noch eine ostdeutsche Regionalpartei, die im Westen - mit Ausnahme des Saarlands eine geringe Organisationsbasis aufweist" (Niedermayer 2019, S. 387), ist, wahrscheinlich wenig ändern.

Allerdings ist zu berücksichtigen, dass in der Linken nicht nur die Gebietsgliederungen und deren Personalstärke entscheidend, sondern hauptsächlich die ideologischen Strömungen ,nach wie vor wesentliche Machtzentren“ (Oppelland und Träger 2012, S. 213) sind. Grundsätzlich lässt sich zwischen den realpolitisch orientierten Reformern (v. a. Forum Demokratischer Sozialismus), dem vielfältig zusammengesetzten Spektrum der Orthodoxen (z. B. Antikapitalistische Linke, Kommunistische Plattform, Sozialistische Linke) und der lagerunabhängigen Emanzipatorischen Linken differenzieren (vgl. Oppelland und Träger 2014, S. 111 ff.). Außerdem kann die Jugendorganisation Linksjugend ['solid] durch 
öffentlichkeitswirksame Aktionen eine wichtige Rolle im innerparteilichen Willensbildungs- und Entscheidungsprozess spielen. In dieser Gemengelage besteht die „Aufgabe der Parteiführung (...) in erster Linie [darin; H.T.], eine Balance zwischen den Strömungen zu wahren“ (Oppelland und Träger 2012, S. 213).

Welche Bedeutung dabei die handelnden Personen haben, lässt sich auch seit der Bundestagswahl 2017 beobachten. Während beispielsweise die Parteivorsitzende Katja Kipping „eine junge, weltoffene und avantgardistische Linke“ (Abé und Deggerich 2017, S. 46) anstrebt, sieht die Sahra Wagenknecht ,in der Flüchtlingspolitik (...) die Hauptursache für die Wählerwanderung von links nach rechts" (ebd.). Dieser Richtungsstreit, der sich hauptsächlich auf die erheblichen Verluste an die AfD zurückführen lässt, bringt Die Linke in ein strategisches Dilemma. Letztlich geht es darum, ob die Partei ihre asyl- und migrationspolitischen Positionen substanziell ändert oder wesentliche Teile ihrer früheren Wählerschaft, die mittlerweile aus Protest gegenüber den etablierten Parteien für die Rechtspopulisten votieren, aufgibt (vgl. Träger 2018b).

In diesem Zusammenhang warfen Wagenknecht und der Dramaturg Bernd Stegemann im Juni 2018 der Linken „Doppelmoral“ (Stegemann und Wagenknecht 2018) vor, denn sie blende

„offensichtliche Zusammenhänge aus, wenn sie ihr eigenes gutes Gefühl in einer Willkommenskultur pflegt, um dann die realen Verteilungskämpfe in ein Milieu zu verbannen, das sich weit weg vom eigenen Leben befindet."

Durch diese öffentliche Kritik erhielt der unmittelbar bevorstehende Bundesparteitag besondere Brisanz. Dort wurde ein Leitantrag des Parteivorstandes, in dem unter anderem ,sichere, legale Fluchtwege, offene Grenzen und ein menschenwürdiges System der Aufnahme von Geflüchteten und ein Lastenausgleich in Europa“ sowie „eine Initiative zur Legalisierung von illegalisierten Menschen“ (Die Linke 2018) gefordert wurden, diskutiert. Letztlich stimmten die Delegierten mit großer Mehrheit der Vorlage zu, nachdem diese um einen Passus hinsichtlich der dezidierten Ablehnung von Abschiebungen ergänzt worden ist. Die Linke bleibt also bei ihren Positionen in der Asyl- und Flüchtlingspolitik und ist damit „das Kontrastprogramm zur AfD“, wie es Katja Kipping (2018b) ausdrückte.

Im weiteren Verlauf des Parteitages kritisierte Wagenknecht (2018a) den innerparteilichen Umgang mit ihr und verlangte „eine solidarische Diskussion“. Anschließend debattierten die Delegierten - abweichend von der Tagesordnung eine Stunde lang über die Rede der Fraktionsvorsitzenden. Kritische Stimmen 
warfen ihrer Genossin vor, ,keine Debatten zuzulassen und die Positionen der Partei zu ignorieren“ (WAZ 2018).

Neben der inhaltlichen Ausrichtung gab es mit der im August 2018 gegründeten Sammlungsbewegung aufstehen einen weiteren Konfliktpunkt innerhalb der Linken. Auch hier spielte Sahra Wagenknecht (2018b, S. 31) die zentrale Rolle, denn sie dachte bereits wenige Monate nach der Bundestagswahl 2017 über ,eine starke linke Volkspartei“ nach. Ein solches Projekt funktioniere allerdings nur, ,wenn prominente Persönlichkeiten mitmachen, die den Menschen die Hoffnung zurückgeben, dass sich politisch etwas in ihrem Sinne bewegt" (ebd.). Dieses Vorhaben stieß in der Partei auf erhebliche Vorbehalte und Widerstand. Die Parteivorsitzende Kipping (2018a, S. 5) warnte vor einer weiteren Spaltung des linken Lagers und schlug vor, ,die Linke weiter als Partei in Bewegung auf[zu]stellen." Einen entsprechenden Beschluss fassten die Delegierten im Juni 2018 auf dem Parteitag. Dem Leitantrag des Parteivorstandes folgend bezeichnete sich Die Linke (2018) als „Partei in Bewegung“" und erklärte, dass darin ,kein Gegensatz“ bestehe.

Unabhängig von der Beschlusslage ihrer Partei bereitete Wagenknecht gemeinsam mit einem Unterstützerkreis, zu dem Politikerinnen und Politiker aus den Reihen von SPD, Linken und Grünen, ${ }^{3}$ Kulturschaffende ${ }^{4}$ sowie Wissenschaftlerinnen und Wissenschaftler ${ }^{5}$ gehörten, ihr Projekt weiter vor (vgl. aufstehen 2018b): Anfang August 2018 schaltete die neue Sammlungsbewegung aufstehen ihren Internetauftritt frei, auf dem sich Interessenten registrieren lassen konnten. Diese Möglichkeit wurde bereits innerhalb der ersten Tage intensiv genutzt (vgl. Feldenkirchen 2018, S. 33), obgleich noch nichts Genaueres über die Initiative bekannt war. Wagenknecht (2018b, S. 25) hatte lediglich ein „Angebot für alle, die mit der herrschenden Politik unzufrieden sind und sich eine Erneuerung des Sozialstaates und eine friedliche Außenpolitik wünschen“, angekündigt. Erst am 4. September 2018 wurde der Gründungsaufruf von aufstehen (2018a) vorgestellt; in dem Papier ist unter anderem von einer neuen Friedenspolitik, sicheren Arbeitsplätzen, naturverträglichem Wirtschaften,

${ }^{3}$ Die überregional bekanntesten sind die Bundestagsabgeordneten Marco Bülow und Sevim Dagdelen, die Kieler Oberbürgermeisterin Simone Lange, der ehemalige Staatsminister im Auswärtigen Amt Ludger Volmer und die frühere Bundestagsvizepräsidentin Antje Vollmer. ${ }^{4}$ Exemplarisch werden Dramaturg Bernd Stegemann, Liedermacher Konstantin Wecker, Sängerin Nina Hagen und Kabarettistin Lisa Fitz genannt.

${ }^{5}$ Darunter ist der Soziologe Wolfgang Streeck, der bis zu seiner Emeritierung das renommierte Max-Planck-Institut für Gesellschaftsforschung leitete. 
„,[e]xzellente[r] Bildung für alle“ und der Rettung der Demokratie die Rede. Konkrete Positionen sollte ein Arbeitsausschuss erarbeiteten.

Die Sammlungsbewegung wurde seitens der Linken kritisiert. Der Parteivorsitzende Bernd Riexinger (zit. nach Abé und Lehmann 2018, S. 43) verwies darauf, dass aufstehen nicht mehr als „140000 Klicks“ sei. Katja Kipping (zit. nach ebd.) erklärte, man könne ,nicht aus einer Ehe heraus die nächste Hochzeit planen.“ Dietmar Bartsch (zit. nach FAZ 2018) gab zu bedenken, ,dass Oskar Lafontaine und Sahra Wagenknecht nicht zwingend für die Zusammenführung von Linken stehen." Benjamin Hoff (zit. ebd.), der die Thüringer Staatskanzlei leitet und dem Reformerlager zuordnet werden kann, bezeichnete die Sammlungsbewegung als ,antiaufklärerisch“. ${ }^{6}$

In den folgenden Monaten kam Sahra Wagenknecht immer mehr in die „Bredouille“ (Decker 2018, S. 2). Dem Projekt aufstehen war letztlich kein öffentlich wahrnehmbarer Erfolg beschieden; die Sammlungsbewegung ,war viel Sammlung, aber wenig Bewegung" (Fried 2019b, S. 4). Zeitweise wurde in der Bundestagsfraktion offen über einen Sturz der Vorsitzenden diskutiert. Dazu kam es allerdings nicht, denn auch Vertreterinnen und Vertreter des Reformerlagers hielten es "für gefährlich, Wagenknecht abzuwählen“ (Abé und Hagen 2018, S. 38). Im Januar 2019 legten die stellvertretende Parteivorsitzende Janine Wissler, die auch die hessische Landtagsfraktion leitet, und der Parlamentarische Geschäftsführer der Bundestagsfraktion, Jan Korte, eine „Strategie gegen die weitere Rechtsverschiebung und gegen die weitere Erosion der Demokratie" (Wissler und Korte 2019) vor. Das Papier, in dem es unter anderem um den Umgang mit der AfD und die damit verbundenen Herausforderungen für Die Linke geht, wurde „als machtpolitische Kampfansage“ (Abé und Hagen 2019, S. 35) wahrgenommen. Zwei Monate später kündigte Sahra Wagenknecht (zit. nach Fried 2019a, S. 5) an, nicht wieder für das Amt der Fraktionsvorsitzenden zu kandidieren, und begründete ihre Entscheidung mit gesundheitlichen Problemen als ,eine[r] direkte[n] Folge des extremen Stresses, den ich in den letzten Jahren hatte." Welche Folgen sich durch diesen Rückzug für aufstehen ergeben, wird sich zeigen. Bernd Stegemann (2019, S. 120), der als Vorstand des Trägervereins

\footnotetext{
${ }^{6} \mathrm{Hoff}$ schrieb in einem mit der damaligen Bundesjustizministerin Katarina Barley (SPD) und dem Bundesgeschäftsführer von Bündnis 90/Die Grünen, Michael Kellner, verfassten Beitrag für den Tagesspiegel: „Wer seine ganze Kraft darauf verwendet, sich selbst und dem linken Lager zu beweisen, der oder die bessere Linke zu sein, vernachlässigt die eigentliche politische Kontroverse mit den Neoliberalen, den Konservativen und den ganz Rechten“ (Barley et al. 2018).
} 
der Sammlungsbewegung fungiert, gab sich zuversichtlich und sagte: „Wagenknecht ist ja nicht weg, sie hat sich nur aus dem Tagesgeschäft zurückgezogen.“

Auch für ihre Partei und Fraktion wird die Entscheidung von Wagenknecht, so umstritten sie in den eigenen Reihen ist, nicht folgenlos bleiben, denn sie ist eine der wenigen „Reizfiguren, um überhaupt noch Aufmerksamkeit für sich zu gewinnen“ (Fried 2019b, S. 4). Als Nachfolgerin wählte die Bundestagsfraktion im November 2019 die weitgehend unbekannte Abgeordnete Amira Mohamed Ali. Diese Personalentscheidung hatte letztlich ,weniger mit der Strahlkraft der Frau aus Niedersachsen zu tun als mit den Machtverhältnissen in der seit langem zerstrittenen Fraktion“ (Wehner 2019, S. 8). Ob Dietmar Bartsch, der bisher oft im Schatten seiner Co-Vorsitzenden stand, die Lücke in der öffentlichen und medialen Wahrnehmung alleine schließen kann, ist unwahrscheinlich.

\section{Die elektorale Ebene: mehr Rückschläge als Lichtblicke}

Angesichts der innerparteilichen Kontroversen und des dadurch entstandenen Eindruckes, Die Linke würde sich hauptsächlich mit sich selbst beschäftigen, kann es kaum verwundern, dass die Partei den ,Sonntagsfragen“ zufolge nicht von der eklatante Schwäche der SPD als der bisher größten Partei im Mitte-Links-Lager profitieren konnte. Seit der Bundestagswahl 2017 oszillierten die Umfragewerte zwischen sieben und elf Prozent. Die Hochphase war jedoch nach einem Jahr vorbei; mittlerweile befindet sich Die Linke allenfalls auf dem Niveau ihres letzten Wahlergebnisses. Zwischenzeitlich sah es so aus, als könnte die Fünf-Prozent-Marke gefährlich nahe kommen (Abb. 3).

Wie weit die Linkssozialisten in den Bereich um die Sperrklausel kommen, wurde bei der Europawahl im Mai 2019 deutlich: Damals kämpfte Die Linke (2019, S. 6) - dem ersten Satz ihres Wahlprogrammes zufolge - ,für ein soziales Europa, für eine andere Europäische Union, in der alle gut arbeiten und leben können." Mit Vorschlägen für ein Verbot von Rüstungsexporten, höhere Löhne, „eine linke Energiepolitik in Europa“ (ebd., S. 33), eine „Mobilität mit weniger Verkehr“ (ebd., S. 34) und einen „Kontinent der Solidarität statt [einer; H.T.] Festung Europa“ (ebd., S. 39) konnte die Partei aber nicht überzeugen. In einer Vorwahlbefragung nannten auf die Frage ,Welche Partei vertritt bei ,Europa“ eine Politik in Ihrem Sinne?“ (Forschungsgruppe Wahlen 2019, S. 22) lediglich fünf Prozent Die Linke; das ist - gleichauf mit der FDP - der niedrigste Wert für alle im Bundestag vertretenen Parteien. Entsprechend schlecht fiel mit 5,5\% das Wahlergebnis aus. Die Sperrklausel, die bei Europawahlen nicht mehr gilt, ist so 


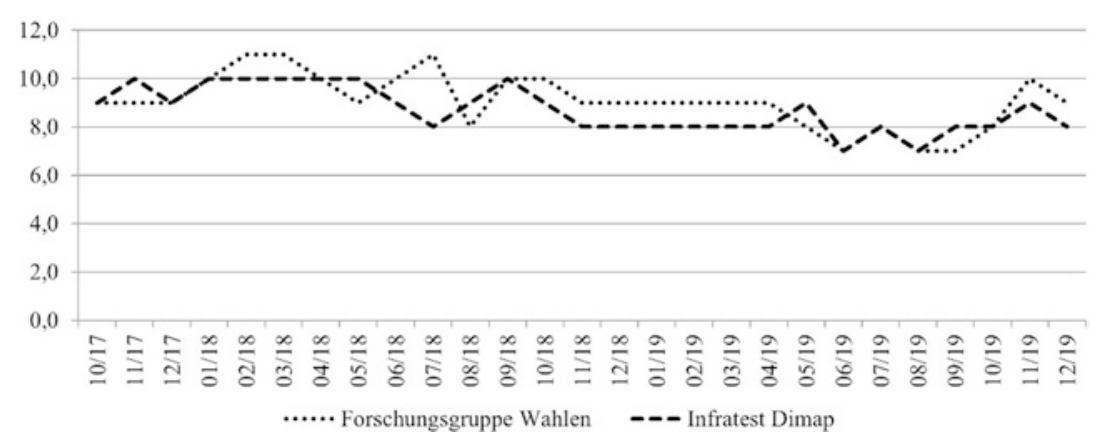

Abb. 3 Die Linke in den ,Sonntagsfragen“ seit der Bundestagswahl 2017 (in Prozent). Für die Monate mit mehreren ,Sonntagsfragen“ ist der Wert der jeweils letzten Umfrage abgebildet. (Quelle: Eigene Darstellung nach Daten von www.wahlrecht.de)

nahe wie seit der Bundestagswahl 2002 nicht mehr; das sollte die Alarmglocken der gesamten Partei schrillen lassen.

Trotz deutlich gestiegener Wahlbeteiligung entfielen auf die Linkssozialisten 112.406 Stimmen weniger als 2014. In absoluten Zahlen stehen die fast flächendeckenden Zuwächse im Westen den erheblichen Verlusten im Osten gegenüber. Aufgrund der höheren Partizipationsquoten sind jedoch überall niedrigere Stimmenanteile zu verbuchen; das gilt vor allem für die neuen Länder und Berlin. Gleichwohl sollte nicht vernachlässigt werden, dass das Wahlergebnis in Ostdeutschland mit 13,4 \% dreieinhalbmal so hoch in Westdeutschland ist. Die Linke hat also ihre Hochburgen immer noch auf dem Gebiet der ehemaligen DDR (Tab. 3).

Ein ambivalentes Bild ergibt sich auch mit Blick auf die sieben Landtagswahlen seit Herbst 2017 (s. Tab. 4): Bei allen vier Abstimmungen im Westen konnte Die Linke deutliche Gewinne verbuchen; gleichwohl blieb sie in Niedersachsen und Bayern außerparlamentarisch (vgl. Meyer und Müller-Rommel 2018; Schultze 2019). In Hessen stellt die Partei trotz der deutlichen Vergrößerung von sechs auf neun Abgeordnete die kleinste der sechs Fraktionen und spielte aufgrund einer fehlenden Mehrheit für Rot-Rot-Grün - genauso wie auf der Bundesebene - keine Rolle bei der Regierungsbildung (vgl. Debus und Faas 2019). Anders gestaltete sich die Situation in Bremen, denn dort konnte Die Linke gemeinsam mit Bündnis 90/Die Grünen die Verluste der SPD so weit ausgleichen, dass ein Bündnis der drei Parteien deutlich auf eine parlamentarische Mehrheit kommt. Der rot-grün-rote Senat, in dem die Linkssozialisten mit 
Tab. 3 Die Linke bei der Europawahl 2019

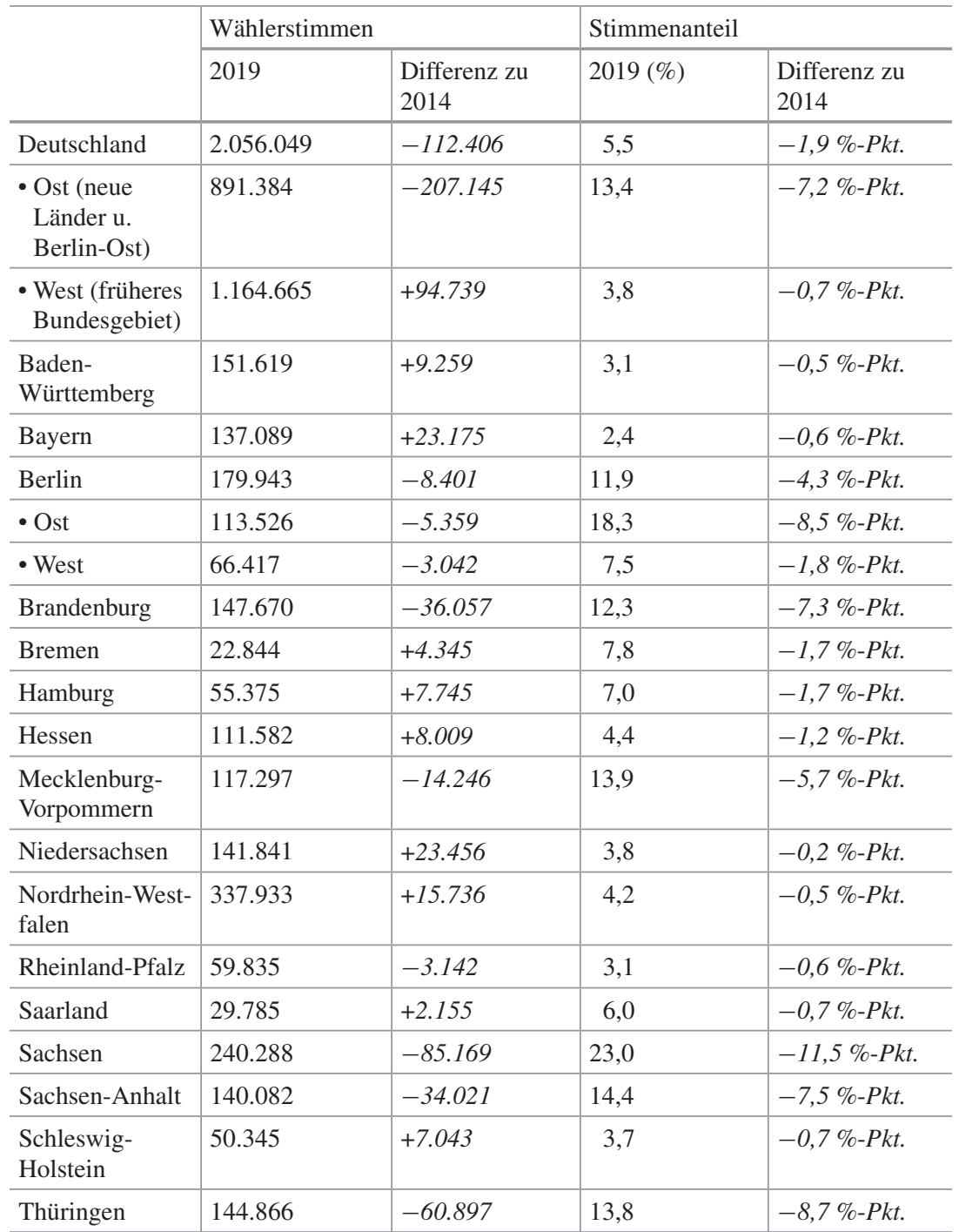

(Quelle: Eigene Darstellung und Berechnungen nach Daten des Bundeswahlleiters (2014, 2019)) 
Tab. 4 Die Linke bei den Landtagswahlen zwischen Oktober 2017 und Oktober 2019

\begin{tabular}{l|l|l|l}
\hline & $\begin{array}{l}\text { Zweitstimmen (oder } \\
\text { Äquivalent) }\end{array}$ & Stimmenanteil & $\begin{array}{l}\text { Wählerwanderung mit } \\
\text { AfD }\end{array}$ \\
\hline $\begin{array}{l}\text { Niedersachsen } \\
\text { (15. Oktober 2017) }\end{array}$ & $\begin{array}{l}177.118 \\
(+64.906)\end{array}$ & $\begin{array}{l}4,6 \% \\
(+1,5 \%-P k t .)\end{array}$ & 10.000 an AfD \\
\hline $\begin{array}{l}\text { Bayern } \\
(14 . \text { Oktober 2018) }\end{array}$ & $\begin{array}{l}437.888 \\
(+186.791)\end{array}$ & $\begin{array}{l}3,2 \% \\
(+1,1 \%-P k t .)\end{array}$ & k. A. \\
\hline $\begin{array}{l}\text { Hessen } \\
\text { (28. Oktober 2018) }\end{array}$ & $\begin{array}{l}181.332 \\
(+19.844)\end{array}$ & $\begin{array}{l}6,3 \% \\
(+1,1 \%-P k t .)\end{array}$ & 15.000 an AfD \\
\hline $\begin{array}{l}\text { Bremen } \\
(26 . \text { Mai 2019) }\end{array}$ & $\begin{array}{l}166.378 \\
(+54.893)\end{array}$ & $\begin{array}{l}11,3 \% \\
(+1,8 \%-P k t .)\end{array}$ & $\begin{array}{l}+/-0 \text { mit AfD } \\
(1000 \text { an CDU) }\end{array}$ \\
\hline $\begin{array}{l}\text { Brandenburg } \\
\text { (1. September 2019) }\end{array}$ & $\begin{array}{l}135.572 \\
(-47.606)\end{array}$ & $\begin{array}{l}10,7 \% \\
(-7,9 \%-P k t .)\end{array}$ & $\begin{array}{l}11.000 \text { an AfD } \\
(19.000 \text { an SPD) })\end{array}$ \\
\hline $\begin{array}{l}\text { Sachsen } \\
\text { (1. September 2019) }\end{array}$ & $(-85.170)$ & $\begin{array}{l}10,4 \% \\
(-8,5 \%-P k t .)\end{array}$ & 26.000 an AfD \\
\hline $\begin{array}{l}\text { Thüringen } \\
\text { (27. Oktober 2019) }\end{array}$ & $\begin{array}{l}343.780 \\
(+78.352)\end{array}$ & $\begin{array}{l}31,0 \% \\
(+2,8 \%-P k t .)\end{array}$ & 16.000 an AfD \\
\hline
\end{tabular}

Anmerkung: Sofern der größte Abwanderungsstrom nicht zur AfD ging, wurde die entsprechende Partei in Klammern verzeichnet.

Quelle: Eigene Darstellung und Berechnungen nach Daten der Landeswahlleiter und der Wahltagsbefragungen von infratest dimap (wahl.tagesschau.de)

Claudia Bernhard und Kristina Vogt die Senatorinnen für Gesundheit, Frauen und Verbraucherschutz sowie für Wirtschaft, Arbeit und Europa stellen, ist seit August 2019 im Amt. Zwölf Jahre nach ihrer Gründung ist Die Linke erstmals in einem westdeutschen Bundesland an einer Regierung beteiligt.

Angesichts der ersten Regierungsbeteiligung der Linken im Westen war es nicht überraschend, dass im Sommer 2019 auch auf Bundesebene wieder über Rot-Rot-Grün (vgl. Bingener 2019; Cleven 2019; Koch 2019) diskutiert wurde. In dieser Situation erlebte die Partei allerdings bei den Wahlen in Brandenburg und Sachsen ein unerwartetes „Desaster“ (Bubrowski 2019, S. 2). Anders als die ,Sonntagsfragen“ vermuten ließen, stürzte Die Linke in beiden Ländern auf Stimmenanteile zwischen zehn und elf Prozentpunkten ab, sodass die Fraktionen so klein wie noch nie seit der Wiedervereinigung sind. In Sachsen haben die Linkssozialisten weiterhin keine Machtoption. In Brandenburg verlor die rotrote Regierung ihre Mehrheit; für Rot-Rot-Grün ist der Mandatsvorsprung mit 45 von 88 Abgeordneten so gering, dass sich die SPD von Ministerpräsident Dietmar Woidke für eine ,Kenia'-Koalition mit CDU und Bündnis 90/Die Grünen 
entschied, sodass Die Linke nach zehn Jahren wieder auf die Oppositionsbänke wechseln musste.

Wirkte sich in Brandenburg und Sachsen das angesichts der ,Sonntagsfragen“ zu erwartende ,Kopf-an-Kopf-Rennen` zwischen der Partei des Ministerpräsidenten und der AfD negativ auf das Wahlergebnis der Linke aus, ist für Thüringen genau das Gegenteil zu beobachten. Der dortige Landesverband konnte ,dank eines präsidial-bürgerlichen und bürgernahen Ramelow, der die Koalition aus Linken, SPD und Grünen fünf Jahre lang sicher durch alle Fährnisse der Landespolitik gelotst hat" (Deckers 2019, S. 1), seinen Stimmenanteil im Vergleich zu 2014 vergrößern und wurde mit 31,0 \% erstmals stärkste politische Kraft in einem Bundesland. Allerdings verlor die Koalition ihre parlamentarische Mehrheit; neben einer rot-rot-grünen Minderheitsregierung könnte Die Linke allenfalls in einem Bündnis mit der AfD oder der CDU regieren. Angesichts der unklaren Mehrheitsverhältnisse im Erfurter Landtag bleibt den Christdemokraten gegenwärtig ,im Grunde keine andere Wahl, als mehr oder weniger offiziell“ (Oppelland 2019) die rot-rot-grüne Koalition, deren Ministerpräsident Ramelow im Februar 2020 überraschend dem FDP-Kandidaten Thomas Kemmerich unterlag und vier Wochen später doch wieder zum Regierungschef gewählt wurde, zu tolerieren. Vor diesem Hintergrund wird sich zeigen, ob es zu ,eine[r] weitere[n] Öffnung der CDU für die weithin sozialdemokratisch auftretenden Linkspartei in Ostdeutschland" (Holzhauser 2019, S. 148) kommt. Mit Blick auf die ,Rote-Socken“-Kampagne, die der damalige CDU-Generalsekretär Peter Hintze 1994 aus Anlass der von der PDS tolerierten rot-grünen Minderheitsregierung in Sachsen-Anhalt entwickelte (vgl. Träger 2011, S. 363 ff.), ist es eine Ironie der Geschichte, dass ausgerechnet die CDU eine Regierung unter Führung der Linken tolerieren muss.

\section{$6 \quad$ Fazit: schwierige Zeiten für zweitkleinste Oppositionsfraktion}

Zum Abschluss des Beitrages werden die vier in der Einleitung herausgearbeiteten Leitfragen aufgegriffen:

- Die Linke ist inzwischen nicht mehr die „erste Adresse der Protestwähler“ (Lang 2018, S. 5), denn sie gehört nach mehreren Regierungsbeteiligungen auf Landesebene und kontinuierlicher Zugehörigkeit zum Bundestag mittlerweile zu den etablierten Parteien. Infolgedessen verliert sie große Teile ihrer früheren Wählerschaft an die AfD, die trotz ihrer Verortung am anderen Ende des politischen Spektrums eine ernstzunehmende Konkurrenz für Die Linke ist. 
- Durch das neue Konkurrenzverhältnis entstand ein innerparteilicher Konflikt über die Ausrichtung in der Asyl- und Integrationspolitik, in dem vor allem Sahra Wagenknecht eine zentrale Rolle spielte. Ausweislich der Wahlergebnisse lässt sich konstatieren, dass Die Linke mit ihrem programmatischen Angebot kaum überzeugen kann. In den gegenwärtig besonders virulenten Politikfeldern - der Zuwanderung und Migration einerseits sowie bei Umweltund Klimaschutz andererseits - haben die Linkssozialistinnen und Linkssozialisten kaum eine Chance. Anders als AfD und Bündnis 90/Die Grünen profitieren sie nicht von der eklatanten Schwäche der Regierungsparteien, sondern sind selbst mit niedrigen Umfragewerte und schlechten Wahlergebnissen konfrontiert. Die Partei trabt also ähnlich wie vor einem Jahrzehnt „,neben oder hinter den Debatten her“ (Neugebauer 2011, S. 167).

- Die öffentliche und mediale Wahrnehmung der Linken wird weiterhin durch die Bundestagsfraktion, die seit 2017 nur noch die zweitkleinste ist und ihren Status als Oppositionsführerin an die AfD verloren hat, geprägt. Das lässt sich insbesondere auf die starke Präsenz von Sahra Wagenknecht zurückführen, die sowohl mit ihren Wortmeldungen als auch mit ihrer Sammlungsbewegung aufstehen das Bild der Partei zeitweise in stärkerem Maße als ihre Vorgänger dominierte. Aber auch nach dem Rückzug Wagenknechts wird die Fraktion eine wichtige Rolle im Kosmos der Linken spielen, denn Dietmar Bartsch ist wesentlich prominierter als die Parteivorsitzenden Katja Kipping und Bernd Riexinger. Die Linke bleibt aller Wahrscheinlichkeit nach eine Fraktionspartei.

- Bei der Bundestagswahl 2017 konnte Die Linke ihren Zweitstimmenanteil auf $9,2 \%$ geringfügig verbessern. Das darf jedoch nicht darüber hinwegtäuschen, dass die Sperrklausel zwischenzeitlich in gefährliche Nähe rückte. Neben den ,Sonntagsfragen', die als Momentaufnahmen ohne längerfristige Aussagekraft ignoriert werden könnten, waren die Europawahl im Mai 2019 sowie die Urnengänge in Brandenburg und Sachsen wenige Monate später ernstzunehmende Alarmsignale für die elektorale Schwäche der Linken. Die Landtagswahl in Thüringen ist wegen des weit über das eigene politische Lager hinaus populären Ministerpräsidenten ${ }^{7}$ eine Ausnahme vom generellen Trend.

\footnotetext{
${ }^{7}$ In der Wahltagsbefragung von infratest dimap (http://wahl.tagesschau.de/wahlen/201910-27-LT-DE-TH/index.shtml) gaben $70 \%$ aller Befragten in Thüringen an, dass Bodo Ramelow „ein guter Ministerpräsident“ sei. Von den Wählerinnen und Wählern der Linken vertraten $99 \%$ diese Meinung; auch bei den Koalitionspartnern waren die Werte mit $95 \%$ (SPD) und $86 \%$ (Bündnis 90/Die Grünen) deutlich überdurchschnittlich hoch. Sogar in der Wählerschaft der Oppositionspartei CDU attestierten $60 \%$ Ramelow gute Arbeit; bei der AfD traf dies lediglich auf $26 \%$ zu.
} 
Ob sich die Partei bis zur regulär im September 2021 stattfindenden Bundestagswahl wieder fangen kann, wird sich zeigen. Gegenwärtig sieht es so aus, als hatten Gero Neugebauer und Richard Stöss (2015, S. 170) Recht, als sie nach der Wahl 2013 davon sprachen, dass Die Linke ,den Zenit ihrer Entwicklung bereits überschritten" habe.

In ihrer gegenwärtigen Verfassung muss Die Linke, so kurios das klingen mag, hoffen, dass die Große Koalition möglichst bis zum turnusgemäßen Ende der Legislaturperiode des Bundestages hält. Vorgezogene Neuwahlen würden die Partei in einer ungünstigen Situation treffen, denn sie leidet als zweitkleinste von sechs Parlamentsfraktionen unter einem „Aufmerksamkeitsdefizit“ (Lang 2018, S. 56), muss den Rückzug von Sahra Wagenknecht aus der ersten Reihe kompensieren und sich programmatisch erkennbarer profilieren. Hinzukommt, dass die SPD im Dezember 2019 mit Saskia Esken und Norbert Walter-Borjans zwei im linken Teil der Sozialdemokratie zu verortende Politiker zu ihren neuen Vorsitzenden wählte und damit in der Linken zumindest kurzzeitig eine Debatte über eine „Fusion mit der SPD“ (Decker 2019) auslöste. Inwieweit die SPD auf der elektoralen Ebene den Linkssozialisten wieder gefährlich werden könnte, wird sich zeigen. Die politischen Akteure der Linken stehen vor einer wahren Herkulesaufgabe, über der das Damoklesschwert einer Bundestagswahl, die - zumindest vor der Covid-19-Pandemie - zwischenzeitlich auch vor dem turnusgemäßen Termin erwartet wurde, schwebt.

\section{Literatur}

Abé, N., \& Deggerich, M. (2017). Souverän geht anders. Der Spiegel, 43, 46.

Abé, N., \& Hagen, K. (2018). Für das Volk. Der Spiegel, 48, 38.

Abé, N., \& Hagen, K. (2019). Zeit für den Neustart. Der Spiegel, 28, 34-35.

Abé, N., \& Lehmann, T. (2018). Frisch geschlüpft. Der Spiegel, 39, 42-43.

Aufstehen. (2018a). Gründungsaufruf. https://www.aufstehen.de/gruendungsaufruf/. Zugegriffen: 13. Sept. 2019.

Aufstehen. (2018b). Unterstützer. https://www.aufstehen.de/unterstuetzer/. Zugegriffen: 13. Sept. 2019.

Bajohr, S. (2017). Die nordrhein-westfälische Landtagswahl vom 14. Mai 2017: Schwarz-Gelb statt Rot-Grün. Zeitschrift für Parlamentsfragen, 48, 614-633. https://doi. org/10.5771/0340-1758-2017-3-614.

Barley, K., Hoff, B., \& Kellner, M. (2018). Wer aufsteht, muss auch vorwärts gehen. https:// causa.tagesspiegel.de/politik/ist-die-zeit-reif-fuer-eine-linke-sammlungsbewegung/weraufsteht-muss-auch-vorwaerts-gehen.html. Zugegriffen: 13. Sept. 2019. 
Bingener, R. (15. August 2019). Hinschauen, offen bleiben. Frankfurter Allgemeine Zeitung, 2.

Bubrowski, H. (3. September 2019). Schlachtfest ohne Schlachteplatte. Frankfurter Allgemeine Zeitung, 2.

Bundeswahlleiter. (2013). Wahl zum 18. Deutschen Bundestag am 22. September 2013. Heft 3: Endgültige Ergebnisse nach Wahlkreisen. https://www.bundeswahlleiter.de/dam/ jcr/a832ae2d-3ffc-4805-92c6-c92cc8668d17/btw13_heft3.pdf. Zugegriffen: 13. Aug. 2019.

Bundeswahlleiter. (2014). Wahl der Abgeordneten des Europäischen Parlaments aus der Bundesrepublik Deutschland am 25. Mai 2014. Heft 3: Endgültige Ergebnisse nach kreisfreien Städten und Landkreisen. https://www.bundeswahlleiter.de/dam/jcr/ ce6da9e1-31a4-4856-9d16-f4baef0d3d6f/ew14_heft3.pdf. Zugegriffen: 13. Aug. 2019.

Bundeswahlleiter. (2017). Wahl zum 19. Deutschen Bundestag am 24. September 2017. Heft 3: Endgültige Ergebnisse nach Wahlkreisen. https://www.bundeswahlleiter.de/dam/ jcr/3f3d42ab-faef-4553-bdf8-ac089b7de86a/btw17_heft3.pdf. Zugegriffen: 13. Aug. 2019.

Bundeswahlleiter. (2019). Europawahl 2019. Heft 3: Endgültige Ergebnisse nach kreisfreien Städten und Landkreisen. https:/www.bundeswahlleiter.de/dam/jcr/5f3be4fff16e-4852-938d-9a038adc29cb/ew19_heft3.pdf. Zugegriffen: 13. Aug. 2019.

Cleven, T. (10./11. August 2019). Grüne reagieren reserviert auf SPD-Avancen. Leipziger Volkszeitung, 2.

Debski, A. (5. September 2019). Nach dem Wahldesaster: Die Linke in Sachsen kämpft ums Überleben. Leipziger Volkszeitung, 1.

Debus, M., \& Faas, T. (2019). Die hessische Landtagswahl vom 28. Oktober 2018: Fortsetzung der schwarz-grünen Wunschehe mit starken Grünen und schwacher CDU. Zeitschrift für Parlamentsfragen, 50, 245-262. https://doi.org/10.5771/0340-17582019-2-245.

Decker, M. (20./21. Oktober 2018). Wagenknecht in der Bredouille. Leipziger Volkszeitung, 2.

Decker, M. (10. Dezember 2019). Linke debattiert über Fusion mit der SPD. Leipziger Volkszeitung, 5.

Deckers, D. (28. Oktober 2019). Ramelow. Frankfurter Allgemeine Zeitung, 1.

Die Linke. (2016). Spitzenteam zur Bundestagswahl 2017. https://www.die-linke.de/partei/ parteistruktur/parteivorstand/2016-2018/beschluesse/detail/news/spitzenteam-zurbundestagswahl-2017/. Zugegriffen: 29. Aug. 2019.

Die Linke. (2017). Sozial. Gerecht. Frieden. Für alle - Die Zukunft, für die wir kämpfen! Wahlprogramm der Partei DIE LINKE zur Bundestagswahl 2017. https://www. die-linke.de/fileadmin/download/wahlen2017/wahlprogramm2017/die_linke_wahlprogramm_2017.pdf. Zugegriffen: 13. Aug. 2019.

Die Linke. (2018). DIE LINKE - Partei in Bewegung. Beschluss der 1. Tagung des 6. Parteitages der Partei DIE LINKE vom 8. bis 10. Juni 2018 in Leipzig. https://www.dielinke.de/start/detail/die-linke-partei-in-bewegung-1/. Zugegriffen: 12. Sept. 2019.

Die Linke. (2019). Europa nur solidarisch. Wahlprogramm der Partei DIE LINKE zur Europawahl 2019. https://www.die-linke.de/fileadmin/download/wahlen2019/wahlprogramm_ pdf/Europawahlprogramm_2019_-_Partei_DIE_LINKE.pdf. Zugegriffen: 13. Aug. 2019.

Ehrenstein, C. (8. Juni 2015). Der Unterhaltungskünstler. Die Welt, S. 6. 
FAZ. (2018). Führende Linkspolitiker grenzen sich von Wagenknecht ab. https://www.faz. net/aktuell/politik/inland/fuehrende-politiker-der-linkspartei-haben-sich-von-wagenknecht-distanziert-15745037.html. Zugegriffen: 13. Aug. 2019.

Feldenkirchen, M. (2018). Was zusammengehört. Der Spiegel, 33, 33.

Fitzpatrick, J. (2018). Zwitschern für Stimmen. Der Einsatz von Twitter im Bundestagswahlkampf 2017. Zeitschrift für Parlamentsfragen, 49, 603-617. https://doi. org/10.5771/0340-1758-2018-3-603.

Forschungsgruppe Wahlen e. V. (2017). Bundestagswahl. Eine Analyse der Wahl vom 24. September 2017 (=Berichte der Forschungsgruppe Wahlen e. V., Mannheim; Nr. 175). Mannheim: Forschungsgruppe Wahlen e. V.

Forschungsgruppe Wahlen e. V. (2019). Europawahl. Eine Analyse der Wahl vom 26. Mai 2019 (=Berichte der Forschungsgruppe Wahlen e. V., Mannheim; Nr. 175). Mannheim: Forschungsgruppe Wahlen e. V.

Fried, N. (12. März 2019a). Aus der Balance. Süddeutsche Zeitung, S. 5.

Fried, N. (12. März 2019b). Rückzug einer Reizfigur. Süddeutsche Zeitung, S. 4.

Hilmer, R., \& Gagné, J. (2018). Die Bundestagswahl 2017: GroKo IV - ohne Alternative für Deutschland. Zeitschrift für Parlamentsfragen, 49, 372-406. https://doi. org/10.5771/0340-1758-2018-2-372.

Holzhauser, T. (2019). Erosion der Abgrenzung? Das Verhältnis der CDU zur Linkspartei in historischer Perspektive. Zeitschrift für Parlamentsfragen, 50, 130-148. https://doi. org/10.5771/0340-1758-2019-1-130.

Hujer, M. (2015). Wandelnder Widerspruch. Der Spiegel, 42, 40-41.

Jesse, E. (2018). Die Bundestagswahl 2017 im Spiegel der repräsentativen Wahlstatistik. Zeitschrift für Parlamentsfragen, 49, 223-242. https://doi.org/10.5771/0340-17582018-2-223.

Jesse, E., \& Lang, J. P. (2012). DIE LINKE - eine gescheiterte Partei? München: Olzog.

Kipping, K. (14./15. April 2018a). „Eine weitere linke Partei spaltet das fortschrittliche Lager“" (Interview). Leipziger Volkszeitung, 5.

Kipping, K. (2018b). Zukunft, wir kommen! (Rede auf dem Leipziger Parteitag). https:// www.die-linke.de/partei/parteistruktur/parteitag/leipziger-parteitag-2018/news-defaultdetailseite/news/zukunft-wir-kommen/. Zugegriffen: 12. Sept. 2019.

Klein, M., Becker, P., Czeczinski, L., Lüdecke, Y., Schmidt, B., \& Springer, F. (2019). Die Sozialstruktur der deutschen Parteimitgliedschaften. Empirische Befunde der Deutschen Parteimitgliederstudien 1998, 2009 und 2017. Zeitschrift für Parlamentsfragen, 50, 81-98. https://doi.org/10.5771/0340-1758-2019-1-81.

Knelangen, W. (2017). Die schleswig-holsteinische Landtagswahl vom 7. Mai 2017: Niederlage der „Küstenkoalition“ und erstmals „Jamaika“ im Norden. Zeitschrift für Parlamentsfragen, 48, 575-593. https://doi.org/10.5771/0340-1758-2017-3-575.

Koch, M. (2. Juli 2019). Die Logik von Rot-Grün-Rot. Leipziger Volkszeitung, 1.

Koschkar, M., \& Nestler, C. (2017). Die mecklenburg-vorpommernsche Landtagswahl vom 4. September 2016: Zäsur des regionalen Parteienwettbewerbs und Fortsetzung der Großen Koalition. Zeitschrift für Parlamentsfragen, 48, 25-39. https://doi. org/10.5771/0340-1758-2017-1-25.

Kosfeld, C.-P. (2015). Zwei neue Oppositionsführer. Das Parlament, 43-45, 12.

Küpper, M. (2. August 2016). Das nationalbolschewistische Kalkül. Frankfurter Allgemeine Zeitung, 4. 
Lafontaine, O. (2016). „Schlechter Stil“ (Interview). Der Spiegel, 16, 30-32.

Lang, J. P. (2018). Abschied vom Osten? Die Linke nach der Bundestagswahl 2017. Sankt Augustin/Berlin: Konrad-Adenauer-Stiftung e.V.

Locke, S., \& Becker, K.B. (28. Oktober 2019). Eine Genugtuung für die Linke. Frankfurter Allgemeine Zeitung, 3.

Meyer, H., \& Müller-Rommel, F. (2018). Die niedersächsische Landtagswahl vom 15. Oktober 2017: „Vernunftheirat“ führt zur ersten Großen Koalition nach 50 Jahren. Zeitschrift für Parlamentsfragen, 49, 3-22. https://doi.org/10.5771/0340-1758-2018-1-3.

Neugebauer, G. (2011). „Quo vadis? Wie die LINKE versucht, sich als Partei und für sich eine Position im Parteiensystem zu finden.“ Interne Konsolidierungsprozesse und Orientierungssuche im Fünf-Parteien-System. In O. Niedermayer (Hrsg.), Die Parteien nach der Bundestagswahl 2009 (S. 157-177). Wiesbaden: VS Verlag. https://doi. org/10.1007/978-3-531-93223-1_7.

Neugebauer, G., \& Stöss, R. (1999). Nach der Bundestagswahl 1998: Die PDS in stabiler Seitenlage? In O. Niedermayer (Hrsg.), Die Parteien nach der Bundestagswahl 1998 (S. 119-140). Opladen: Leske + Budrich.

Neugebauer, G., \& Stöss, R. (2003). Die PDS in Not. In O. Niedermayer (Hrsg.), Die Parteien nach der Bundestagswahl 2002 (S. 125-158). Opladen: Leske + Budrich.

Neugebauer, G., \& Stöss, R. (2007). Die Partei Die Linke. Nach der Gründung in des Kaisers neuen Kleidern? Eine politische Bedarfsgemeinschaft als neue Partei im deutschen Parteiensystem. In O. Niedermayer (Hrsg.), Die Parteien nach der Bundestagswahl 2005 (S. 151-199). Wiesbaden: VS Verlag.

Neugebauer, G., \& Stöss, R. (2015). Den Zenit überschritten: Die Linkspartei nach der Bundestagswahl 2013. In O. Niedermayer (Hrsg.), Die Parteien nach der Bundestagswahl 2013 (S. 159-173). Wiesbaden: Springer VS. https://doi.org/10.1007/978-3-65802852-7_7.

Niedermayer, O. (2015). Die brandenburgische Landtagswahl vom 14. September 2014: Die Linke wird abgestraft, bleibt aber Regierungspartei. Zeitschrift für Parlamentsfragen, 46, 21-38.

Niedermayer, O. (2016). Parteimitgliedschaften im Jahre 2015. Zeitschrift für Parlamentsfragen, 47, 411-436. https://doi.org/10.5771/0340-1758-2016-2-411.

Niedermayer, O. (2017a). Die Berliner Abgeordnetenhauswahl vom 18. September 2016: Zersplitterung des Parteiensystems und halber Machtwechsel. Zeitschrift für Parlamentsfragen, 48, 40-56. https://doi.org/10.5771/0340-1758-2017-1-40.

Niedermayer, O. (2017b). Parteimitgliedschaften im Jahre 2016. Zeitschrift für Parlamentsfragen, 48, 370-396. https://doi.org/10.5771/0340-1758-2017-2-370.

Niedermayer, O. (2018a). Die Entwicklung des deutschen Parteiensystems. Zur Bedeutung kurzfristiger Faktoren im Jahrzehnt des europäischen Wandels. Zeitschrift für Parlamentsfragen, 49, 286-303. https://doi.org/10.5771/0340-1758-2018-2-286.

Niedermayer, O. (2018b). Parteimitgliedschaften im Jahre 2017. Zeitschrift für Parlamentsfragen, 49, 346-371. https://doi.org/10.5771/0340-1758-2018-2-346.

Niedermayer, O. (2019). Parteimitgliedschaften im Jahre 2018. Zeitschrift für Parlamentsfragen, 50, 385-410. https://doi.org/10.5771/0340-1758-2019-2-385.

Oppelland, T. (2015). Die thüringische Landtagswahl vom 14. September 2014: Startschuss zum Experiment einer rot-rot-grünen Koalition unter linker Führung. Zeitschrift für Parlamentsfragen, 46, 39-56. 
Oppelland, T. (2019). Thüringen vor der Landtagswahl vom 27. Oktober 2019. https:// regierungsforschung.de/wp-content/uploads/2019/09/16092019_regierungsforschung. de_Oppelland_Wahl-Th\%C3\%BCringen.pdf. Zugegriffen: 18. Sept. 2019.

Oppelland, T., \& Träger, H. (2012). DIE LINKE: Die Macht der Strömungen. In K.-R. Korte \& J. Treibel (Hrsg.), Wie entscheiden Parteien? Prozesse innerparteilicher Willensbildung in Deutschland (S. 189-217). Baden-Baden: Nomos.

Oppelland, T., \& Träger, H. (2014). Die Linke. Willensbildung in einer ideologisch zerstrittenen Partei. Baden-Baden: Nomos.

Oppelland, T., \& Träger, H. (2016). Ein neuer Koalitionstyp: Voraussetzungen für rot-rote bzw. rot-rot-grüne Koalitionen unter Führung der Linken auf Landesebene. Zeitschrift für Politik, 63, 24-44. https://doi.org/10.5771/0044-3360-2016-1-24.

Schultze, R.-O. (2019). Die bayerische Landtagswahl vom 14. Oktober 2018: Signal für nachhaltigen Wandel auch im Bund? Zeitschrift für Parlamentsfragen, 50, 223-244. https://doi.org/10.5771/0340-1758-2019-2-223.

Schüttemeyer, S. S., \& Höhne, B. (2019). Durch das Nadelöhr der Demokratie. Die Kandidatenaufstellung der Partei DIE LINKE zur Bundestagswahl 2017. Berlin: Institut für Parlamentsforschung.

Spier, T. (2016). Die Linke. Von der Regionalpartei Ost zur Partei des sozialen Souterrains. In E. Wiesendahl (Hrsg.), Parteien und soziale Ungleichheit (S. 191-221). Wiesbaden: Springer VS.

Stegemann, B., \& Wagenknecht, S. (2018). Von linker Moral und neoliberalen Interessen. https://www.zeit.de/2018/24/linke-sammlungsbewegung-sahra-wagenknechtpopulismus/komplettansicht. Zugegriffen: 12. Sept. 2019.

Stegemann, B. (2019). „Auf diesem Dampfer steuert die Linke in den Untergang“ (Interview). Der Spiegel, 13, 118-120.

Träger, H. (2011). Die ostdeutschen Landesverbände in den Bundesparteien. Personelle, inhaltlich-programmatische und finanzielle Beziehungen (1990-2007). Frankfurt a. M.: Peter Lang.

Träger, H. (2015). Ein Vierteljahrhundert Wahlen in Ost und West (1990 bis 2014): regionale Unterschiede und Gemeinsamkeiten. Zeitschrift für Parlamentsfragen, 46, $57-81$.

Träger, H. (2018a). Die Bundestagwahl 2017 in Ostdeutschland: ein Alarmsignal für die Volksparteien. Gesellschaft - Wirtschaft - Politik, 67, 295-301. https://doi.org/10.3224/ gwp.v67i3.02.

Träger, H. (2018b). Neue innerparteiliche Konflikte in der LINKEN und Wagenknechts Bewegung aufstehen. https://regierungsforschung.de/neue-innerparteiliche-konflikte-inder-linken-und-wagenknechts-bewegung-aufstehen/. Zugegriffen: 12. Aug. 2019.

Wagenknecht, S. (2016). „Merkel verantwortet schlimmsten Rechtsruck nach 1945“ (Interview). https://www.welt.de/politik/deutschland/article153550410/Merkel-verantwortetschlimmsten-Rechtsruck-nach-1945.html. Zugegriffen: 29. Aug. 2019.

Wagenknecht, S. (2017). Wir wollen soziale Gerechtigkeit, wir wollen Frieden (Parteitagsrede). https://www.die-linke.de/partei/parteistruktur/parteitag/hannoverscher-parteitag-2017/redenund-grussworte/news/wir-wollen-soziale-gerechtigkeit-wir-wollen-frieden/. Zugegriffen: 29. Aug. 2019.

Wagenknecht, S. (2018a). Dieses Land verändern (Rede auf dem Leipziger Bundesparteitag). https://www.die-linke.de/start/detail/dieses-land-veraendern/. Zugegriffen: 12. Sept. 2019. 
Wagenknecht, S. (2018b). „Ich wünsche mir eine linke Volkspartei“ (Interview). Der Spiegel, 3 (25).

WAZ. (2018). Nach Wagenknecht-Rede eskaliert Linken-Streit über Asyl. https://www. waz.de/politik/nach-wagenknecht-rede-eskaliert-linken-streit-ueber-asyl-id214533403. html. Zugegriffen: 12. Sept. 2019.

Wehner, M. (13. November 2019). Linke Blitzkarriere. Frankfurter Allgemeine Zeitung, 8.

Wiedmann-Schmidt, W. (2016a). Die Zwangsehe. Der Spiegel, 22, 34-37.

Wiedmann-Schmidt, W. (2016b). Volkes Stimme. Der Spiegel, 31, 38.

Wiedmann-Schmidt, W. (2016c). Wundern über Sahra. Der Spiegel, 13, 32.

Winkler, J. R. (2018). Die saarländische Landtagswahl vom 26. März 2017: Bestätigung der CDU-geführten Großen Koalition. Zeitschrift für Parlamentsfragen, 50, 40-56. https://doi.org/10.5771/0340-1758-2018-1-40.1.

Wissler, J., \& Korte, J. (2019). Die Kämpfe verbinden. https://www.linksfraktion.de/ themen/nachrichten/detail/die-kaempfe-verbinden/. Zugegriffen: 18. Sept. 2019.

Wyssuwa, M. (30. Mai 2016). Eine Torte schließt die Reihen. Frankfurter Allgemeine Zeitung, 2. 